\title{
Expression of PAX2 in papillary serous carcinoma of the ovary: immunohistochemical evidence of fallopian tube or secondary Müllerian system origin?
}

\author{
Guo-Xia Tong ${ }^{1}$, Luis Chiriboga ${ }^{2}$, Diane Hamele-Bena ${ }^{1}$ and Alain C Borczuk ${ }^{1}$ \\ ${ }^{1}$ Department of Pathology, Columbia University Medical Center, New York, NY, USA and \\ ${ }^{2}$ Department of Pathology, New York University Medical Center, New York, NY, USA
}

\begin{abstract}
PAX2 is a urogenital developmental transcription factor expressed in the Wolffian ducts, developing kidneys, and Müllerian ducts during embryonic stage. Its function in renal development is well documented and its clinical application in the diagnosis of lesions of renal origin has been reported recently. However, information on its role in the Müllerian-derived genital tract is sparse. In this study, we investigated the expression of PAX2 in human female genital tract using immunohistochemistry. We demonstrated that PAX2 was expressed specifically in the epithelial cells of fallopian tube, endometrial and endocervical glands, but not in the stromal tissues in these areas. PAX2 was detected in secondary Müllerian structures in the ovary, such as endometriotic and endosalpingiotic glands and rete ovarii, but not in ovarian surface epithelium, surface epithelium-derived inclusion cysts, stroma, or sex-cord-derived structures such as follicles, oocytes, and corpus luteum. In addition, PAX2 was detected in $67 \%$ of ovarian papillary serous carcinomas $(N=36)$ but rarely in peritoneal malignant mesotheliomas, with two exceptions $(N=54)$. Interestingly, the two PAX2-positive 'peritoneal malignant mesotheliomas' were from female patients and were positive for estrogen receptor. The significance of expression of PAX2 and estrogen receptor in these cases is under investigation. Taken together, we suggest that PAX2 is a novel Müllerian-specific epithelial marker when used in proper clinical settings. Identification of PAX2 in the majority of papillary serous carcinomas of the ovary but not in the ovarian surface epithelium or epithelium-derived inclusion cysts suggests that this malignant epithelial tumor may be directly derived from the primary or secondary Müllerian epithelium in or surrounding the ovary, rather than from the surface epithelium or its derivatives.
\end{abstract}

Modern Pathology (2007) 20, 856-863; doi:10.1038/modpathol.3800827; published online 25 May 2007

Keywords: PAX2; human Müllerian system; female genital tract; papillary serous carcinoma of the ovary; peritoneal malignant mesothelioma

Papillary serous carcinoma of the ovary is one of the most common and lethal malignant tumors. It constitutes $60 \%$ of the malignant tumors of the ovary and is the leading cause of death from gynecologic malignancies. ${ }^{1}$ Despite the clinical significance and extensive research, the pathogenesis of this tumor is still a matter of debate. The purported tissue origins include the ovarian surface epithelium, fallopian tube, and secondary Müllerian system, with the surface epithelial origin

Correspondence: Dr G-X Tong, MD, PhD, Department of Pathology, Columbia University Medical Center, 630 W 168th street, PH1564-D, New York, NY 10032, USA.

E-mail: gt2125@columbia.edu

Received 19 January 2007; revised 05 April 2007; accepted 09 April 2007; published online 25 May 2007 as the dominant theory in this field. ${ }^{2,3}$ However, the evidence for each theory is largely circumstantial. Progress in this area is hindered by the advanced stage of this tumor at the time of diagnosis and the absence of specific markers for Müllerian epithelium.

PAX2 is a member of the pair box (PAX) gene family, which consists of nine members, each encoding a nuclear transcription factor, PAX1PAX9. ${ }^{4}$ These transcription factors are expressed in a spatial and temporal pattern during embryogenesis and are implicated in the control of organogenesis in several systems. ${ }^{5}$ PAX2 is specifically expressed in the developing central nervous system, eye, ear, and urogenital tract and is essential for the development of these organs. In early developmental stages, PAX2 is first detected in the intermediate mesoderm, from 
which the Wolffian duct and the kidneys derive. PAX2 is expressed in Wolffian ducts and all stages of developing kidneys. ${ }^{6}$ The significance of PAX2 in renal development has been confirmed both in experimental animal models and in patients with spontaneous PAX2 mutations. ${ }^{7-10}$ In addition, PAX2 is detected in renal cell carcinomas, ${ }^{11}$ Wilms' tumors, ${ }^{12,13}$ and nephrogenic adenomas ${ }^{14,15}$ and is proposed to be a specific marker in identification of lesions of renal origin.

The Müllerian ducts also originate from the intermediate mesoderm adjacent to the Wolffian ducts and give rise to the female genital tract, including fallopian tubes, uterus, cervix, and the upper portion of vagina. As in the Wolffian tubes and the developing kidneys, ${ }^{16,17}$ PAX2 is detected in the Müllerian ducts and its derivatives in mice. ${ }^{18}$ However, information on the function and the clinical significance of PAX2 in the human Müllerian system is sparse ${ }^{19,20}$ in contrast to what is known about its role in renal development. In this study, we utilized immunohistochemistry (IHC) to investigate the expression of PAX2 in the fallopian tubes, uterus, cervix, and ovaries of adult women, as well as papillary serous carcinomas of the ovary. We found that PAX2 was specifically expressed in the epithelial cells of the female genital tract from the fimbriae ends to the cervix, and in papillary serous carcinomas of the ovary. We suggest that PAX2 can be used as a specific epithelial marker for tumors of Müllerian origin. Additionally, we think that finding of PAX2 expression in papillary serous carcinomas might provide new insight into the histogenesis of this ovarian epithelial tumor.

\section{Materials and methods}

The fallopian tube $(N=7)$, uterus $(N=6)$, cervix $(N=5)$, and the ovaries $(N=10)$ used in this study were from 15 women from 22 to 58 years of age and were resected for benign lesions such as endometriosis, uterine prolapse, leiomyomata, or sterilization.

A total of 36 papillary serous carcinomas of the ovary, 54 malignant mesotheliomas of the peritoneum, and eight specimens from hernioplasties or omentectomies (for normal mesothelial controls) were retrieved from archival material from the Department of Pathology at the Columbia University Medical Center from 1997 to 2004. Five tissue microarrays (MTA) were constructed and composed of 3 to 4 cores of $1 \mathrm{~mm}$ in diameter from each case. The 36 papillary serous carcinomas included 34 cases in which the ovaries were the primary sites of tumors and two cases in which the ovaries were involved by papillary serous carcinomas of the uterus and fallopian tube. Patients' ages varied from 45 to 80 years, with a mean age of 62 years. Of these 36 cases, six were classified as low-grade and the rest as high-grade papillary serous carcinomas. The diagnoses of these ovarian papillary serous carcinomas were established by the clinical information and histologic examination, with rare cases requiring immunohistochemical confirmation. The 54 malignant mesotheliomas (45 epithelioid, eight biphasic, and one sarcomatous) were predominantly located in the omentum and peritoneum, and included 37 male and 17 female patients. The male patients ranged in ages from 31 to 75 (mean age 54) years and the female patients from 15 to 67 (mean age 49) years. The diagnoses of malignant mesothelioma of the peritoneum were made based on clinical information and histologic examination, and were confirmed by immunohistochemical stains including calretinin, WT-1, CEA, LeuM1, BerEP4, B72.3, and CK5/6.

IHC for PAX2, as well as calretinin, estrogen receptor (ER), and progesterone receptor (PR) were performed in this study, utilizing the avidin-biotin peroxidase method in a DAKO AutoStainer (Carpinteria, CA, USA). In brief, the paraffin-embedded MTA and conventional tissue sections were deparaffinized and incubated with $3 \%$ hydrogen peroxide for $15-20 \mathrm{~min}$ to block endogenous peroxidase activity. After antigen retrieval treatment, the sections were sequentially incubated with the primary antibodies, the biotinylated secondary antibody, the avidin-biotin complex, and the chromogenic substrate $3,3^{\prime}$-diaminobenzidine, and counterstained with hematoxylin.

PAX2, ER, and PR are nuclear proteins and have distinct nuclear staining patterns. Calretinin has both cytoplasmic and nuclear staining. These immunohistochemical stains were reviewed by two pathologists and were graded semiquantitatively according to the intensity of nuclear reactivity as negative, weak $(1+)$, moderate $(2+)$, and strong $(3+)$. The percentage of positive cells $(5-20 \%$, $25-80 \%$, and $100 \%$ ) was also recorded.

\section{Results}

Expression of PAX2 in the Fallopian Tubes, Uterus, Cervix, and Vaginal Cuff

As shown in Figure 1a-d, PAX2 was detected in the epithelial cells of the fallopian tube, endometrial and cervical glands. The ciliated and secretory cells in the intraluminal epithelium and the epithelium in the fimbrial end of the fallopian tubes were strongly positive for PAX2. Of interest, it was noted that in the fimbriae there was a pattern of focally alternating PAX2-positive and -negative cells (Figure 1a and b). In contrast, the epithelial cells in the endometrial glands were uniformly and continuously positive for PAX2. The glands in proliferative endometrium appeared to have a higher level of PAX2 staining than the glands in secretory endometrium and lower uterine segments (Figure 1c-f). The mucinous glands in the cervix were PAX2 positive and appeared to have lower 

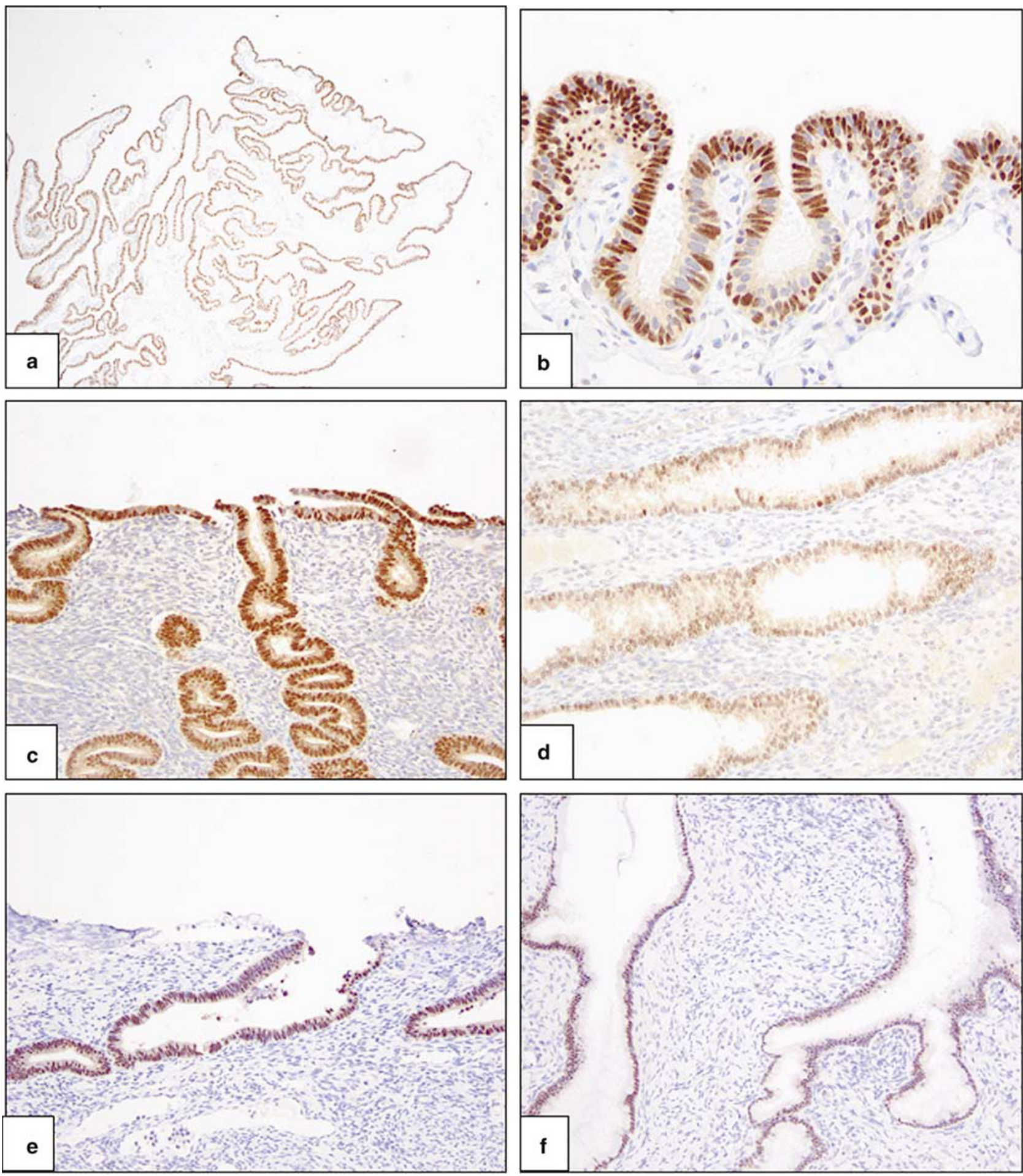

Figure 1 Expression of PAX2 in the female genital tract. PAX2 was detected in the epithelial cells of the female genital tract, but not in the stromal tissue in these areas. (a) Fallopian tube, fimbriae $(\times 40)$. (b) Fallopian tube, fimbriae $(\times 400)$. (c) Proliferative endometrium $(\times 200)$. (d) Secretory endometrium $(\times 200)$. (e) Lower uterine segment $(\times 200)$. (f) The endocervix $(\times 200)$.

levels of PAX2 than the fallopian tubes and proliferative endometrium. The squamous cells in the cervix and the vaginal cuff were negative for PAX2 (data not shown). No PAX2 was detected in the endosalpingeal, endometrial, or endocervical stroma (Figure 1a-f).

\section{Expression of PAX2 in the Ovary}

In most of the cases, the ovarian surface epithelium appears cuboidal or flattened. In one of the ovaries, there was focally papillary hyperplasia of the surface epithelium. The surface 
epithelial cells, including the hyperplastic surface papillae, were negative for PAX2, but positive for calretinin, consistent with their mesothelial origin (Figure 2a-c).

Approximately 30 inclusion cysts derived from the ovarian surface epithelium were noted in these ovaries, and were usually identified in the subcortical areas as elongated or collapsed tubular structures lined by flattened cells. Some showed a continuum with the surface epithelium. These inclusion cysts were also positive for calretinin, but negative for PAX2 (Figure 2d-f). No PAX2 was detected in the ovarian stroma (Figure $2 \mathrm{~b}, \mathrm{e}, \mathrm{h}$, $\mathrm{k}, \mathrm{n}$ ) or in sex-cord-derived structures such as follicles, oocytes, and corpus luteum (data not shown).

However, there were several types of tubular/ glandular structures in the ovary that were positive for PAX2 PAX2 was detected in endometriotic glands, which were surrounded by typical endometrial-type stroma (Figure 2g, h). PAX2 was also detected in the tubules and glands scattered in the loose fibrovascular tissue of the hilar areas (Figure $2 \mathrm{j}$, k). These structures were negative for ER and $\mathrm{PR}$, and consistent with rete ovarii. ${ }^{21-23} \mathrm{In}$ addition, PAX2 was detected in isolated small round glands in the cortical areas; these were lined with columnar epithelial cells and were devoid of recognizable endometrial stroma. These cells were morphologically similar to the secretory cells of the fallopian tubes and were positive for ER and PR, suggestive of endosalpingiotic glands or remnants of the distal Müllerian tubes (Figure 2m, n). All these PAX2 positive structures were negative for calretinin (Figure 2i, l, o).

\section{Expression of PAX2 in Papillary Serous Carcinomas of the Ovary and Malignant Mesotheliomas of the Peritoneum}

PAX2 was detected in $24(67 \%)$ of the 36 papillary serous carcinomas of the ovary. The number of PAX2-positive tumor cells varied from 5 to $100 \%$, with $5-20 \%, 25-80 \%$, and $100 \%$ in 11,9 , and 4 cases, respectively (Figure 3). Staining intensity was graded as weak, moderate, and strong in 4, 9, and 11 cases, respectively, with most cases (20/24) showing moderate or strong nuclear staining (Table 1). There is no significant correlation of tumor grade and PAX2 expression. Three of the six low-grade papillary serous carcinoma was positive for PAX2 expression with diffuse strong staining in two cases and focal weak stain in one. While nine of 24 high-grade papillary serous carcinomas were negative for PAX2. PAX2 was detected in both papillary and solid components of the tumor. ER and PR were detected in $33(92 \%)$ and 15 $(42 \%)$ of 36 papillary serous carcinomas of the ovary, respectively. All the papillary serous carcinomas were negative for calretinin.
No PAX2, ER, or PR was detected in the 37 malignant mesotheliomas of the peritoneum in males. However, two of the 17 cases of malignant mesotheliomas of the peritoneum in females stained positive PAX2. Interestingly, these two PAX2-positive cases were also positive for ER, but not for PR. An additional malignant mesothelioma of the peritoneum from a 15-year-old girl was positive for PR, but negative for ER and PAX2.

\section{Discussion}

As a morphogenic transcription factor, PAX2 is involved in the developmental control of urogenital system, eye, ear, and midbrain. The function of PAX2 in renal development is well established. Mice with heterozygous deletion of PAX2 show underdeveloped kidneys and ureters, while mice with homozygous deletion of PAX2 genes lack kidneys and ureters and die shortly after birth. ${ }^{6}$ Patients with spontaneous mutations of PAX2 have been identified in several families and show the signs and symptoms of so-called renal-coloboma syndrome, including renal hypoplasia, vesicoureteric reflux, and optic nerve malformation. ${ }^{24,25}$ However, the function of PAX2 in the rest of the urogenital system, particularly the Müllerian-ductderived female genital tract is almost unknown. In this study, we investigated the expression of PAX2 in the genital tract of adult women with IHC. We found that PAX2 was expressed in the epithelial cells of the entire Müllerian-duct-derived female genital tract, from the fimbrial ends of the fallopian tubes to the mucinous epithelial cells of endocervical glands, with the exception of the squamous epithelial cells in the cervix and vagina. No PAX2 was detected in the stromal tissue in these sites. The level of PAX2 expression varied depending on the anatomic site and functional status of the female genital epithelium. The epithelial cells in the fallopian tubes and proliferative endometrial glands had higher levels of PAX2 than those in the lower uterine segment, secretory endometrial glands, and endocervical glands, suggesting that PAX2 might be subject to the regulation of steroid hormones. The level of PAX2 in various parts of female genital tract may reflect varying responsiveness to steroid hormones.

The ovary itself is not a Müllerian-derived organ; however secondary Müllerian structures including the remnants of Müllerian ducts, rete ovarii, and glands of endometriosis, endosalpingiosis, and endocervicosis are frequently found in the ovary. ${ }^{26}$ In this study, ovarian surface epithelial cells were negative for PAX2 but positive for calretinin, consistent with their mesothelial origin. PAX2 was not detected in the ovarian stroma or sexcord-derived structures such as follicles, oocytes, and corpus lutea. Ovarian surface epitheliumderived, calretinin-positive inclusion cysts $^{27}$ were 

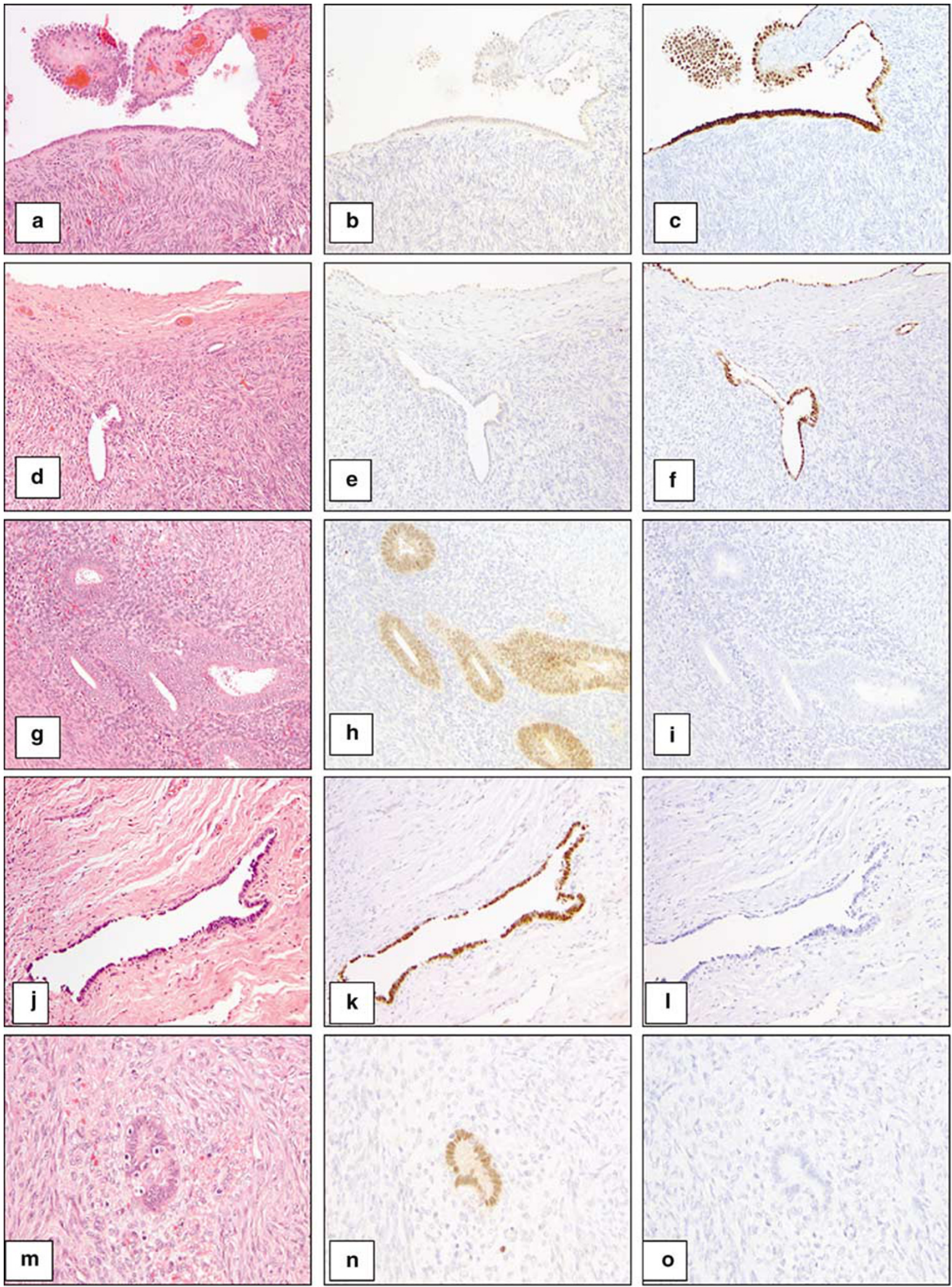

Figure 2 Expression of PAX2 in the ovary. PAX2 was detected in the glands of endometriosis, endosalpingiosis, and rete ovarii in the ovary, but not in the ovarian stroma, surface epithelium, or surface epithelium-derived surface papilloma and inclusion cysts. (a-c) The surface epithelium with focal papillary hyperplasia, H\&E, PAX2, and calretinin, respectively $(\times 100)$. (d-f) The surface epithelium and inclusion cysts, H\&E, PAX2, and calretinin, respectively $(\times 100)$. (g-i) Endometriosis, H\&E, PAX2, and calretinin, respectively $(\times 100)$. (j-l) Rete ovarii, H\&E, PAX2, and calretinin, respectively $(\times 100)$. $(\mathbf{m}-\mathbf{o})$ Endosalpingiosis, H\&E, PAX2, and calretinin, respectively $(\times 100)$. 


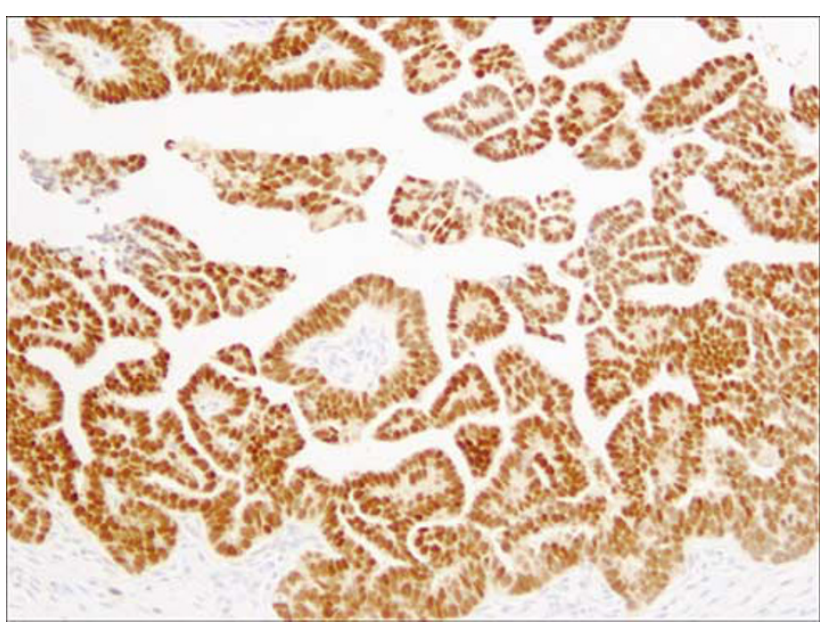

Figure 3 Expression of PAX2 in papillary serous carcinoma of the ovary. A representative case was diffusely positive for PAX2 $(\times 200)$

Table 1 Summary of PAX2 immunohistochemical staining in 24 cases of ovarian PSC

$\%$ of PAX2-positive cells

Staining intensity

\begin{tabular}{lccc}
\cline { 2 - 4 } & Weak & Moderate & Strong \\
\hline $5-20$ & 3 cases & 6 cases & 2 cases \\
$25-80$ & 1 case & 2 cases & 6 cases \\
100 & 0 cases & 1 case & 3 cases \\
\hline
\end{tabular}

also negative for PAX2. However, PAX2 was specifically detected in glands of endometriosis, endosalpingiosis, and rete ovarii. In summary, we demonstrated that PAX2 was not only present in primary Müllerian epithelium, but also in the secondary Müllerian structures and we therefore propose that PAX2 can be used as an immunohistochemical marker to identify these structures.

Papillary serous carcinoma of the ovary is the most common malignant tumor of the ovary. Morphologically, the tumor cells resemble the serous cells of the fallopian tube and are indistinguishable from similar malignant serous tumors in the fallopian tube and the uterus. It has been a dominant theory that the ovarian surface epithelium or the surface epithelium-derived inclusion cysts in the ovary are the tissues of origin for papillary serous carcinoma of the ovary. However, despite extensive effort, the evidence to support this theory is still circumstantial. The ovarian surface epithelium is in continuum with the mesothelium of pelvis and is similar to the mesothelium at morphological, immunohistochemical, and ultrastructural levels. However, papillary serous carcinomas of the ovary rarely show the immunohistochemical or ultrastructural features of malignant mesothelioma.
In addition, searches for premalignant lesions for papillary serous carcinoma in the surface epithelium and in inclusion cysts have proven to be futile. Recently, the clinical practice of prophylactic salpingoophorectomy for women with BRCA1 and BRCA2 mutations revived the interest in alterative theories, such as fallopian tube origin or origin from the secondary Müllerian system.

In addition to the risk of developing breast cancer, women with BRCA1 or BRCA2 have significantly increased risks of developing ovarian or fallopian tube carcinomas, most frequently of the papillary serous type. ${ }^{28-30}$ Thorough examinations of the prophylactic salpingoophorectomy specimens offer the opportunity to search for premalignant lesions for papillary serous carcinoma. Occult malignant lesions have been reported in $2-17 \%$ of these specimens. Although papillary serous carcinoma occurs more often in the ovary than in fallopian tubes clinically, the early premalignant lesions were more frequently identified in the fallopian tubes. ${ }^{31-33}$ It is proposed that the fallopian tube, particularly the fimbria, is the most common location for early serous carcinoma. ${ }^{34}$ In the present study, we demonstrated that a significant number (67\%) of ovarian PSC were focally or diffusely positive for PAX2 and that PAX2 was detected in fallopian tubes and secondary Müllerian structures in the ovary, but not in the ovarian surface epithelium or inclusion cysts. Therefore, our findings are in favor of the fallopian tube or the secondary Müllerian system in the ovary being the origin of ovarian papillary serous carcinoma.

The differential diagnosis of papillary serous carcinomas of the ovary and malignant mesotheliomas of the peritoneum is important clinically because of their differences in the response to chemo/radiation therapy and the association with occupational asbestos exposure. Histologic examination alone may not be conclusive and ancillary tests such as immunohistochemistry are often requested.$^{35}$ Immunohistochemical panels are commonly used to distinguish these two entities. However, the utility of these markers in pelvic/ abdominal tumors is not as great as in the pleural cavity when they are used to differentiate lung adenocarcinomas from mesotheliomas. For example, except calretinin and Ber-EP4, markers such as podoplanin, thrombomodulin, cytokeratin $5 / 6$, WT1, B72.3, CD15, CEA, and CA19-9 are not helpful in the differential diagnosis of papillary serous carcinoma and malignant mesothelioma of the peritoneum due to the decreased sensitivity and/or specificity. ${ }^{36,37}$ Recently, ER was reported to be positive in $93 \%$ of papillary serous carcinoma of the ovary and the peritoneum and PR was positive in $29 \%$ of these tumors. In contrast, ER and PR were reportedly negative in all malignant mesotheliomas of the peritoneum. ${ }^{38,39}$ In this study, ER and PR were detected in 92 and $42 \%$ of papillary serous carcinomas of the ovary, respectively, similar to reported 
findings. No ER or PR was detected in the 37 malignant mesotheliomas of the peritoneum in males that we studied. However, ER was detected in two $(11.8 \%)$ and $\mathrm{PR}$ in one $(5.9 \%)$ of 17 of malignant mesotheliomas of the peritoneum in females in our study. Interestingly, PAX2 was also detected in these two ER-positive peritoneal mesotheliomas, but not in the PR-positive case in which the patient was a teenage girl. Whether this was due to aberrant expression of $\mathrm{PAX}^{40-42}$ in malignant tumors, or Müllerian metaplastic transformation of malignant mesotheliomas, ${ }^{43,44}$ is under further investigation. Nevertheless, PAX2 might be useful as an additional immunohistochemical marker in the differential diagnosis of papillary serous carcinomas of the ovary and female malignant mesotheliomas of the peritoneum, with moderate sensitivity (67\%) and specificity (88\%).

In conclusion, we demonstrated PAX2 expression in Müllerian-derived human female genital tract epithelium and the secondary Müllerian system in the ovary. PAX2 may therefore be considered as a Müllerian lineage epithelial marker in addition to its roles as a marker of renal- and Wolffian duct-derived tissues. Our findings that most papillary serous carcinomas of the ovary were positive for PAX2, but ovarian surface epithelium and the surface epithelium-derived inclusion cysts were not suggests that the ovarian epithelial tumor might be directly derived from primary Müllerian-derived structures (such as fallopian tubes or the fimbriae) or secondary Müllerian structures present in the ovary or its vicinity. Finally, as a lineage transcription factor, PAX2 might be useful in the differential diagnosis of Müllerian-derived tumors in the proper clinical settings.

\section{References}

1 Heintz A, Odicino F, Maisonneuve P, et al. Carcinoma of the ovary. Int J Gynaecol Obstet 2006;95(Suppl 1): S161-S192.

2 Dubeau L. The cell of origin of ovarian epithelial tumors and the ovarian surface epithelium dogma: does the emperor have no clothes? Gynecol Oncol 1999;72:437-442.

3 Piek JM, Kenemans P, Verheijen RH. Intraperitoneal serous adenocarcinoma: a critical appraisal of three hypotheses on its cause. Am J Obstet Gynecol 2004;191:718-732.

4 Gruss P, Walther C. Pax in development. Cell 1992;69:719-722.

5 Lang D, Powell SK, Plummer RS, et al. PAX genes: roles in development, pathophysiology, and cancer. Biochem Pharmacol 2007;73:1-14.

6 Torres M, Gomez-Pardo E, Dressler GR, et al. Pax-2 controls multiple steps of urogenital development. Development 1995;121:4057-4065.

7 Lechner MS, Dressler GR. Mapping of Pax-2 transcription activation domains. Mech Dev 1997;62: 105-120.
8 Eccles MR. The role of PAX2 in normal and abnormal development of the urinary tract. Pediatr Nephrol 1998;12:712-720.

9 Eccles MR, He S, Legge M, et al. PAX genes in development and disease: the role of PAX2 in urogenital tract development. Int J Dev Biol 2002;46:535-544.

10 Dressler GR, Woolf AS. Pax2 in development and renal disease. Int J Dev Biol 1999;43:463-468.

11 Daniel L, Lechevallier E, Giorgi R, et al. Pax-2 expression in adult renal tumors. Hum Pathol 2001;32: 282-287.

12 Li CM, Guo M, Borczuk A, et al. Gene expression in Wilms' tumor mimics the earliest committed stage in the metanephric mesenchymal-epithelial transition. Am J Pathol 2002;160:2181-2190.

13 Dressler GR, Douglass EC. Pax-2 is a DNA-binding protein expressed in embryonic kidney and Wilms' tumor. Proc Natl Acad Sci USA 1992;89:1179-1183.

14 Mazal PR, Schaufler R, Altenhuber-Muller R, et al. Derivation of nephrogenic adenomas from renal tubular cells in kidney-transplant recipients. N Engl J Med 2002;347:653-659.

15 Tong GX, Melamed J, Mansukhani M, et al. PAX2: a reliable marker for nephrogenic adenoma. Mod Pathol 2006;19:356-363.

16 Oefelein M, Grapey D, Schaeffer T, et al. Pax-2: a developmental gene constitutively expressed in the mouse epididymis and ductus deferens. J Urol 1996;156:1204-1207.

17 Kuschert S, Rowitch DH, Haenig B, et al. Characterization of Pax-2 regulatory sequences that direct transgene expression in the Wolffian duct and its derivatives. Dev Biol 2001;229:128-140.

18 Fickenscher HR, Chalepakis G, Gruss P. Murine Pax-2 protein is a sequence-specific trans-activator with expression in the genital system. DNA Cell Biol 1993;12:381-391.

$19 \mathrm{Wu} \mathrm{H}$, Chen Y, Liang J, et al. Hypomethylation-linked activation of PAX2 mediates tamoxifen-stimulated endometrial carcinogenesis. Nature 2005;438:981-987.

20 Shang Y. Molecular mechanisms of oestrogen and SERMs in endometrial carcinogenesis. Nat Rev Cancer 2006;6:360-368.

21 Devouassoux-Shisheboran M, Silver SA, Tavassoli FA. Wolffian adnexal tumor, so-called female adnexal tumor of probable Wolffian origin (FATWO): immunohistochemical evidence in support of a Wolffian origin. Hum Pathol 1999;30:856-863.

22 Nogales FF. Mesohephric (Wolffian) tumors of the female genital tract: is mesonephric histogenesis a mirage and trap? Curr Diagn Pathol 1995;2:94-100.

23 Khan MS, Dodson AR, Heatley MK. Ki-67, oestrogen receptor, and progesterone receptor proteins in the human rete ovarii and in endometriosis. J Clin Pathol 1999;52:517-520.

24 Sanyanusin P, McNoe LA, Sullivan MJ, et al. Mutation of PAX2 in two siblings with renal-coloboma syndrome. Hum Mol Genet 1995;4:2183-2184.

25 Sanyanusin P, Schimmenti LA, McNoe LA, et al. Mutation of the PAX2 gene in a family with optic nerve colobomas, renal anomalies and vesicoureteral reflux. Nat Genet 1995;9:358-364.

26 Lauchlan SC. The secondary Müllerian system revisited. Int J Gynecol Pathol 1994;13:73-79.

27 Cao QJ, Jones JG, Li M. Expression of calretinin in human ovary, testis, and ovarian sex cord-stromal tumors. Int J Gynecol Pathol 2001;20:346-352. 
28 Meeuwissen PA, Seynaeve C, Brekelmans CT, et al. Outcome of surveillance and prophylactic salpingooophorectomy in asymptomatic women at high risk for ovarian cancer. Gynecol Oncol 2005;97:476-482.

29 Olivier RI, van Beurden M, Lubsen MA, et al. Clinical outcome of prophylactic oophorectomy in BRCA1/ BRCA2 mutation carriers and events during follow-up. Br J Cancer 2004;90:1492-1497.

30 Lee Y, Medeiros F, Kindelberger D, et al. Advances in the recognition of tubal intraepithelial carcinoma: applications to cancer screening and the pathogenesis of ovarian cancer. Adv Anat Pathol 2006;13:1-7.

31 Powell CB, Kenley E, Chen LM, et al. Risk-reducing salpingo-oophorectomy in BRCA mutation carriers: role of serial sectioning in the detection of occult malignancy. J Clin Oncol 2005;23:127-132.

32 Tok EC, Ertunc D, Tataroglu C, et al. Clinicopathologic study of the putative precursor lesions of epithelial ovarian cancer in low-risk women. Int J Gynecol Cancer 2006;16:501-508.

33 Leeper K, Garcia R, Swisher E, et al. Pathologic findings in prophylactic oophorectomy specimens in high-risk women. Gynecol Oncol 2002;87:52-56.

34 Medeiros F, Muto MG, Lee Y, et al. The tubal fimbria is a preferred site for early adenocarcinoma in women with familial ovarian cancer syndrome. Am J Surg Pathol 2006;30:230-236.

35 Baker PM, Clement PB, Young RH. Malignant peritoneal mesothelioma in women: a study of 75 cases with emphasis on their morphologic spectrum and differential diagnosis. Am J Clin Pathol 2005;123: 724-737.

36 Ordonez N. Value of immunohistochemistry in distinguishing peritoneal mesothelioma from serous carcinoma of the ovary and peritoneum: a review and update. Adv Anat Pathol 2006;13:16-25.
37 Attanoos RL, Webb R, Dojcinov SD, et al. Value of mesothelial and epithelial antibodies in distinguishing diffuse peritoneal mesothelioma in females from serous papillary carcinoma of the ovary and peritoneum. Histopathology 2002;40:237-244.

38 Barnetson RJ, Burnett RA, Downie I, et al. Immunohistochemical analysis of peritoneal mesothelioma and primary and secondary serous carcinoma of the peritoneum: antibodies to estrogen and progesterone receptors are useful. Am J Clin Pathol 2006;125:67-76.

39 Ordonez NG. Value of estrogen and progesterone receptor immunostaining in distinguishing between peritoneal mesotheliomas and serous carcinomas. Hum Pathol 2005;36:1163-1167.

40 Silberstein GB, Dressler GR, Van Horn K. Expression of the PAX2 oncogene in human breast cancer and its role in progesterone-dependent mammary growth. Oncogene 2002;21:1009-1016.

41 Khoubehi B, Kessling AM, Adshead JM, et al. Expression of the developmental and oncogenic PAX2 gene in human prostate cancer. J Urol 2001;165:2115-2120.

42 Muratovska A, Zhou C, He S, et al. Paired-Box genes are frequently expressed in cancer and often required for cancer cell survival. Oncogene 2003;22:7989-7997.

43 Sawh RN, Malpica A, Deavers MT, et al. Benign cystic mesothelioma of the peritoneum: a clinicopathologic study of 17 cases and immunohistochemical analysis of estrogen and progesterone receptor status. Hum Pathol 2003;34:369-374.

44 Nakayama K, Masuzawa $\mathrm{H}$, Li SF, et al. Immunohistochemical analysis of the peritoneum adjacent to endometriotic lesions using antibodies for Ber-EP4 antigen, estrogen receptors, and progesterone receptors: implication of peritoneal metaplasia in the pathogenesis of endometriosis. Int J Gynecol Pathol 1994;13:348-358. 